\title{
Orthostatic hypotension after cervicomedullary junction surgery: illustrative case
}

\author{
Kasper S. Jacobsen, MD, ${ }^{1,2}$ Rico F. Schou, MD, ${ }^{3}$ Frantz R. Poulsen, MD, PhD, ${ }^{1,2}$ and Christian B. Pedersen, $M D^{1,2}$ \\ ${ }^{1}$ Department of Neurosurgery, Odense University Hospital, Southern Denmark, Denmark; ${ }^{2}$ BRIDGE (Brain Research-Interdisciplinary Guided Excellence), Department of \\ Clinical Research, University of Southern Denmark, Odense, Denmark; and ${ }^{3}$ Department of Anesthesiology and Intensive Care, Odense University Hospital, Southern Denmark, \\ Denmark
}

\begin{abstract}
BACKGROUND Surgery at the cervicomedullary junction carries a risk of damaging vital brainstem functions. Because the nucleus of the solitary tract (NS) is involved in the baroreceptor reflex, damage to its integrity may lead to orthostatic hypotension.

OBSERVATIONS A 56-year-old man with a medical history of hypertension, von Hippel-Lindau disease, and previous bilateral adrenalectomy due to pheochromocytoma was referred with symptoms of dysphagia and paralysis of the left vocal cord. Paralysis of the left vagus nerve was suspected. Magnetic resonance imaging revealed a contrast-enhancing cystic process in the cervicomedullary junction. Twenty-three years earlier, the patient had undergone surgical treatment for a hemangioblastoma in the same region. After repeated surgery, the patient temporarily developed orthostatic hypotension. At discharge, the patient no longer needed antihypertensive medication.
\end{abstract}

LESSONS Surgery near the cervicomedullary junction can affect the NS, leading to disruption of the baroreceptor response that regulates blood pressure.

https://thejns.org/doi/abs/10.3171/CASE21448

KEYWORDS cervicomedullary junction; orthostatic hypotension; fossa posterior; hemangioblastoma; nucleus tractus solitarius; pheochromocytoma

Von Hippel-Lindau disease increases the risk of pheochromocytoma and hemangioblastoma. We review a case of a 56-year-old man who developed orthostatic hypotension after surgery for a hemangioblastoma in the cervicomedullary junction. Orthostatic hypotension can be acute or chronic, and there are several therapeutic possibilities. ${ }^{1-3}$ This illustrative case presents the treatment of complicating postoperative hypotension in the intensive care unit.

\section{Illustrative Case}

A 56-year-old man was referred to our hospital after 1 month of dysphagia. Laryngoscopy revealed paralysis of the left vocal cord, and paralysis of the vagus nerve was suspected. The patient was incapable of swallowing food or medication and had received a nasogastric tube before hospital admission. The patient had a medical history of von Hippel-Lindau disease with multiple tumors in the central nervous system as well as adrenal pheochromocytoma leading to previous bilateral adrenalectomy and substitutional treatment with a glucocorticoid (hydrocortisone) $10 \mathrm{mg}$ twice daily. In addition, the patient was treated with a low-dose calcium antagonist (amlodipine $5 \mathrm{mg}$ daily) for hypertension. Twenty-three years before referral, the patient had undergone surgery for hemangioblastoma in the posterior fossa, and postoperatively he received a ventriculoperitoneal shunt. Three years before referral, the patient had undergone tumor surgery for a hemangioblastoma at the thoracolumbar junction of the spinal canal, and 1 year before referral, the patient had undergone craniotomy in the left temporal region for the removal of a World Health Organization grade I meningioma.

After admission, magnetic resonance imaging (MRI) revealed a contrast-enhancing tumor with a cystic component measuring $3.1 \times 2.2 \times$ $2.0 \mathrm{~cm}\left(\sim 6.8 \mathrm{~cm}^{3}\right)$ that severely compressed the medulla oblongata near the midline and a known contrast-enhancing tumor at the pituitary stalk (Figs. 1 and 2). Subacute surgery was planned to decompress the brainstem, whereas the suprasellar tumor was left intact because blood samples had revealed normal function of the pituitary gland.

Immediately before surgery, tumor-feeding vessels from the right anterior inferior cerebellar artery and posterior inferior cerebellar

ABBREVIATIONS MRI = magnetic resonance imaging; NE = norepinephrine; NS = nucleus of the solitary tract. INCLUDE WHEN CITING Published November 15, 2021; DOI: 10.3171/CASE21448.

SUBMITTED August 10, 2021. ACCEPTED September 9, 2021.

(C) 2021 The authors, CC BY-NC-ND 4.0 (http://creativecommons.org/licenses/by-nc-nd/4.0/). 


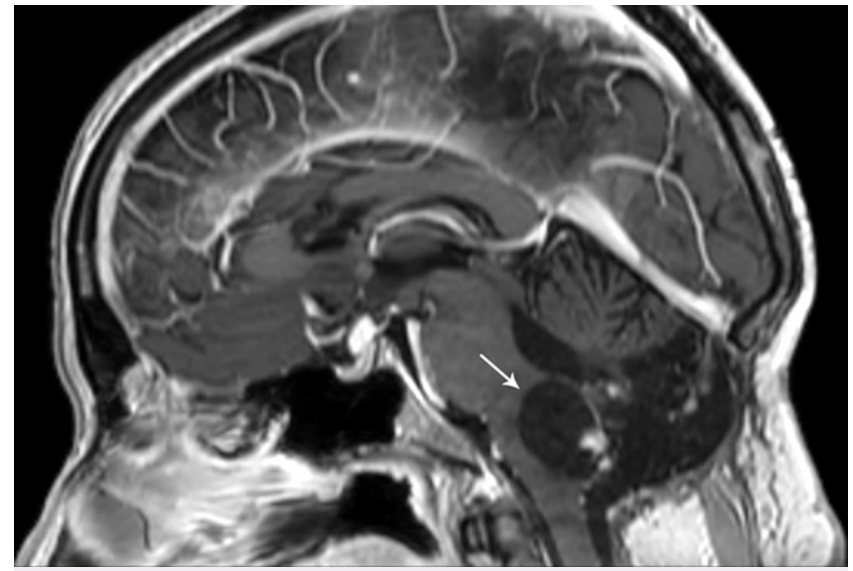

FIG. 1. Presurgical sagittal, gadolinium contrast-enhanced, T1weighted MRI. The white arrow points to the primary cystic process with contrast-enhancing elements.

artery were embolized. The patient was then placed in the prone position with flexion of the neck. The procedure was complicated by previous surgery because of scarred arachnoid tissue and difficulties distinguishing pathological tissue from normal tissue. After surgery, the patient was extubated without new neurological deficits.

The planned postsurgical MRI revealed that the cyst compressing the brainstem was unchanged, and repeated surgery was scheduled. During the second surgery, the cyst was localized and fenestrated. Further exploration was discouraged because the patient had several events of severe bradycardia during surgery. The following day, the patient was fully awake and without new neurological

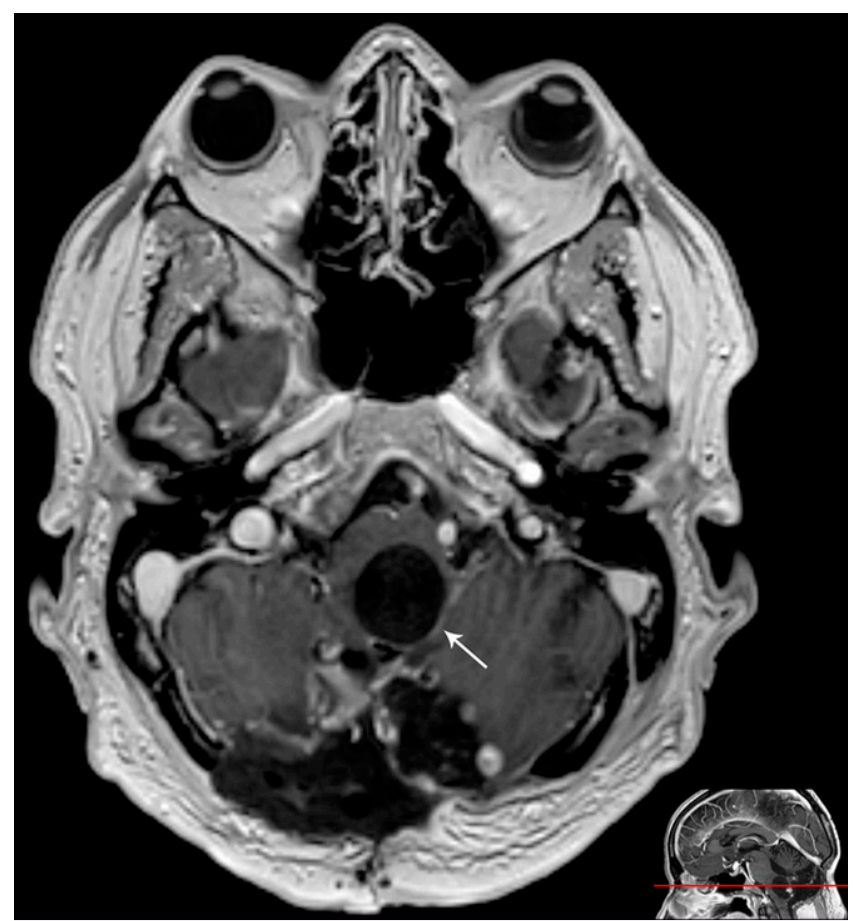

FIG. 2. Presurgical axial, gadolinium contrast-enhanced, T1-weighted MRI. The white arrow points to the primary cystic process.

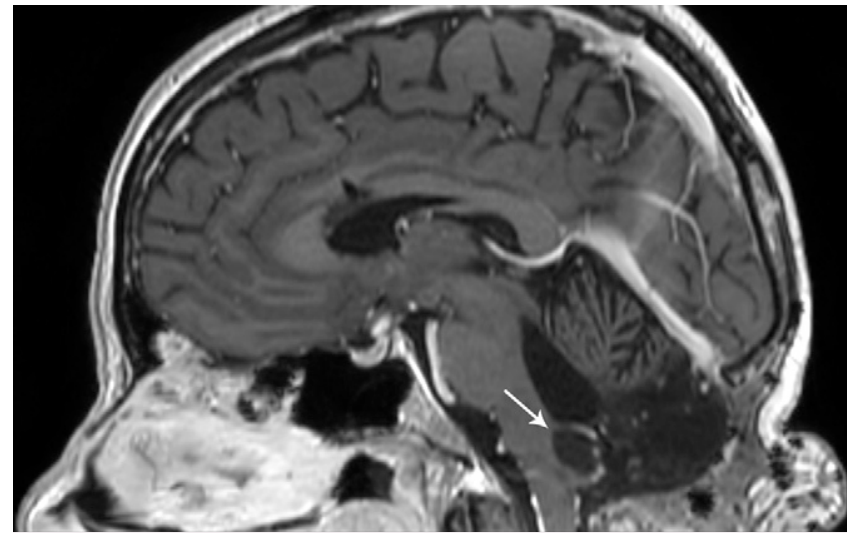

FIG. 3. Sagittal, gadolinium contrast-enhanced, T1-weighted MRI 9 months after surgery. The white arrow points to the residual cystic component that has decreased since the operation.

symptoms, but he remained intubated due to dysphagia and pneumonia. Three days after surgery, the patient could be extubated. Laryngoscopy 5 days after surgery revealed unchanged paralysis of the left vocal cords.

During the early postoperative days, the patient had several episodes of hypotension with systolic blood pressure $<80 \mathrm{~mm} \mathrm{Hg}$ and mean arterial pressure $<50 \mathrm{~mm} \mathrm{Hg}$ without antihypertensive medication. His heart rate was unaffected. He experienced dizziness and looked pale. A change of position from supine to resting on either side could trigger a need for increased norepinephrine (NE), but most episodes occurred during attempts at mobilization. The NE doses required ranged from $0.02 \mu \mathrm{g} / \mathrm{kg} / \mathrm{min}$ to $0.10 \mu \mathrm{g} / \mathrm{kg} / \mathrm{min}$. The synthetic mineralocorticoid with glucocorticoid effect (fludrocortisone acetate, Florinef) reduced the need for NE. Fluid and electrolyte balance was closely monitored, and care was taken that the patient did not become hypovolemic. The episodes with orthostatic hypotension lasted 9 days from the last surgical procedure. At the time of discharge, the patient's blood pressure levels ranged from 100 to $124 \mathrm{~mm} \mathrm{Hg}$ systolic and from 70 to $72 \mathrm{~mm} \mathrm{Hg}$ diastolic without antihypertensive medication.

At discharge, the patient still received hydrocortisone $20 \mathrm{mg}$ three times daily with planned tapering in the following weeks. MRI performed 9 months after surgery showed a considerably smaller cystic tumor measuring $1.8 \times 1.2 \times 0.8 \mathrm{~cm}\left(\sim 0.9 \mathrm{~cm}^{3}\right)$ (Fig. 3).

\section{Discussion \\ Observations}

This patient developed orthostatic hypotension after surgery for hemangioblastoma at the cervicomedullary junction. The patient was receiving medical treatment for hypertension at admission, but treatment was no longer necessary at discharge after surgery. In this case, blood pressure control was further disrupted by previous bilateral adrenalectomy and substitutional hydrocortisone treatment.

Differential diagnostics such as sepsis, arrhythmia, shock, pulmonary embolism, heart failure, hypovolemia, and adrenal insufficiency were excluded. Manipulation in the proximity of the nucleus of the solitary tract (NS) in the dorsomedial medulla during tumor surgery was the most likely explanation for the postoperative orthostatic hypotension. 
The NS is part of the baroreceptor reflex. Mechanoreceptor signals from the carotid sinus and aortic arc are mediated via the carotid sinus nerve (Hering's nerve), a branch of the glossopharyngeal nerve (cranial nerve IX), and via the vagus nerve (cranial nerve $\mathrm{X}$ ). Signals from the chemoreceptors and other visceral organs reach the NS as well. The NS relays information to the caudal ventrolateral medulla, which affects the rostral ventrolateral medulla, where sympathetic activity is generated. The NS also relays information to the dorsal nucleus of the vagus nerve and ambiguous nucleus, where parasympathetic activity is generated. When blood pressure decreases, the sympathetic activity increases and releases NE, which primarily stimulates blood vessel contractility and, to a lesser extent, cardiac output. At the same time, parasympathetic activity to the heart decreases, leading to increased heart rate. ${ }^{2-4}$ The combined result is increased blood pressure.

Several case reports ${ }^{5-14}$ and a review ${ }^{15}$ have described that disruption of the NS and its efferent fibers can cause baroreceptor failure. Overall, the reports show a poor clinical outcome, with death in 5 out of 14 cases, and varying effect of compressive garments, volume expansions, mineralocorticoids, and sympathomimetics.

The patient described in this case experienced only temporary orthostatic hypotension. However, the presurgical antihypertensive treatment could be discontinued permanently at discharge. Therefore, it is likely that the patient became normotensive due to partial disruption of the brainstem functions mentioned above.

\section{Lessons}

Surgery near the cervicomedullary junction can affect the NS, leading to disruption of the baroreceptor response that regulates blood pressure.

\section{Acknowledgments}

We thank the patient for allowing us to write and publish this case; Claire Gudex, Department of Clinical Research, University of Southern Denmark, for proofreading; and the Department of Radiology, University of Southern Denmark, for supplying imagery.

\section{References}

1. Ali A, Ali NS, Waqas N, et al. Management of orthostatic hypotension: a literature review. Cureus. 2018;10(8):e3166.

2. Furlan R, Magatelli R, Palazzolo L, Rimoldi A, Colombo S, Porta A. Orthostatic intolerance: different abnormalities in the neural sympathetic response to a gravitational stimulus. Auton Neurosci. 2001;90(1-2):83-88.

3. Biaggioni I, Shibao CA, Diedrich A, Muldowney JAS 3rd, Laffer CL, Jordan J. Blood pressure management in afferent baroreflex failure: JACC review topic of the week. J Am Coll Cardiol. 2019;74(23): 2939-2947.

4. Freeman R, Abuzinadah AR, Gibbons C, Jones P, Miglis MG, Sinn DI. Orthostatic hypotension: JACC state-of-the-art review. J Am Coll Cardiol. 2018;72(11):1294-1309.
5. Gómez-Esteban JC, Berganzo K, Tijero B, Barcena J, Zarranz JJ. Orthostatic hypotension associated with an epidermoid tumor of the IV ventricle. J Neurol. 2009;256(8):1357-1359.

6. Hocker S, Hoover JM, Puffer RC, Meyer FB. Orthostatic hypotension following resection of a dorsal medullary hemangioblastoma. Neurocrit Care. 2012;16(2):306-310.

7. Hsu CY, Hogan EL, Wingfield W Jr, et al. Orthostatic hypotension with brainstem tumors. Neurology. 1984;34(9):1137-1143.

8. Idiaquez J, Araya P, Benarroch E. Orthostatic hypotension associated with dorsal medullary cavernous angioma. Acta Neurol Scand. 2009;119(1):45-48.

9. Jabary NS, Sarabia R, Sanchez T, Gordillo R. Midodrine treatment in the management of severe orthostatic hypotension after hemangioblastoma surgery. Acta Neurochir (Wien). 2007;149(3):303-306.

10. O'Malley WE, O'Doherty DS, Auth TL. Orthostatic hypotension as a manifestation of posterior fossa tumor. Dis Nerv Syst. 1970;31(12): 846-850.

11. Riedel G, Frewin DB, Gladstone L, Downey JA. Orthostatic hypotension following surgery on brain-stem neoplasms: report of two cases. Arch Phys Med Rehabil. 1974;55(10):471-473.

12. Tadros R, Tonkin A. Bilateral bulbar palsy and postural hypotension following surgery for fourth ventricle subependymoma. J Clin Neurosci. 2009;16(12):1646-1648.

13. Telerman-Toppet N, Vanderhaeghen JJ, Warszawski M. Orthostatic hypotension with lower brain stem glioma. J Neurol Neurosurg Psychiatry. 1982;45(12):1147-1150.

14. Yamashita A, Hirayama M, Koike Y, Nukata M, Hashizume M, Takahashi A. Orthostatic hypotension caused by a localised dorsal medullary tumour. J Neurol Neurosurg Psychiatry. 1996;61(1): 118-119.

15. Nangunoori R, Koduri S, Singh A, Aziz K. Refractory orthostatic hypotension caused by a recurrent hemangioblastoma: case report and review of the literature. Cureus. 2016;8(3):e540.

\section{Disclosures}

The authors report no conflict of interest concerning the materials or methods used in this study or the findings specified in this paper.

\section{Author Contributions}

Conception and design: Jacobsen, Poulsen. Acquisition of data: Jacobsen. Analysis and interpretation of data: Jacobsen, Poulsen. Drafting the article: all authors. Critically revising the article: Jacobsen, Schou, Poulsen. Reviewed submitted version of manuscript: all authors. Approved the final version of the manuscript on behalf of all authors: Jacobsen. Administrative/technical/material support: Jacobsen, Pedersen.

\section{Correspondence}

Kasper S. Jacobsen: Odense University Hospital, Southern Denmark, Denmark. kasper.jacobsen@rsyd.dk. 